University of Wollongong

Research Online

Faculty of Science - Papers (Archive) Faculty of Science, Medicine and Health

$1-1-2009$

\title{
Multiresonator-based Chipless RFID System for Low-Cost Item Tracking
}

Stevan Preradovic

Monash University

Isaac Balbin

Monash University

Nemai Karmakar

Monash University

Gerhard F. Swiegers

University of Wollongong, swiegers@uow.edu.au

Follow this and additional works at: https://ro.uow.edu.au/scipapers

Part of the Life Sciences Commons, Physical Sciences and Mathematics Commons, and the Social and Behavioral Sciences Commons

\section{Recommended Citation}

Preradovic, Stevan; Balbin, Isaac; Karmakar, Nemai; and Swiegers, Gerhard F.: Multiresonator-based Chipless RFID System for Low-Cost Item Tracking 2009, 1411-1419.

https://ro.uow.edu.au/scipapers/346 


\title{
Multiresonator-based Chipless RFID System for Low-Cost Item Tracking
}

\begin{abstract}
A fully passive printable chipless RFID system is presented. The chipless tag uses the amplitude and phase of the spectral signature of a multiresonator circuit and provides I: 1 correspondence of data bits. The tag comprises of a microstrip spiral multiresonator and cross-polarized transmitting and receiving microstrip ultra-wideband disc loaded monopole antennas. The reader antenna is a log periodic dipole antenna with average 5.5-dBi gain. Firstly, a 6-bit chipless tag is designed to encode 000000 and 010101 IDs. Finally, a 35-bit chipless tag based on the same principle is presented. The tag has potentials for lowcost item tagging such as banknotes and secured documents.
\end{abstract}

\section{Keywords}

chipless, cost, rfid, item, multiresonator, tracking, system, low

Disciplines

Life Sciences | Physical Sciences and Mathematics | Social and Behavioral Sciences

Publication Details

Preradovic, S., Balbin, I., Karmakar, N. \& Swiegers, G. F. (2009). Multiresonator-based Chipless RFID System for Low-Cost Item Tracking. IEEE Transactions on Microwave Theory and Techniques, 57 (5), 1411-1419. 


\title{
Multiresonator-Based Chipless RFID System for Low-Cost Item Tracking
}

\author{
Stevan Preradovic, Student Member, IEEE, Isaac Balbin, Student Member, IEEE, \\ Nemai Chandra Karmakar, Senior Member, IEEE, and Gerhard F. Swiegers
}

\begin{abstract}
A fully passive printable chipless RFID system is presented. The chipless tag uses the amplitude and phase of the spectral signature of a multiresonator circuit and provides $1: 1$ correspondence of data bits. The tag comprises of a microstrip spiral multiresonator and cross-polarized transmitting and receiving microstrip ultra-wideband disc loaded monopole antennas. The reader antenna is a log periodic dipole antenna with average 5.5-dBi gain. Firstly, a 6-bit chipless tag is designed to encode 000000 and 010101 IDs. Finally, a 35-bit chipless tag based on the same principle is presented. The tag has potentials for low-cost item tagging such as banknotes and secured documents.
\end{abstract}

Index Terms-Antenna, chipless RF identification (RFID), crosspolarization, multiresonator, reader, transponder, ultra-wideband (UWB).

\section{INTRODUCTION}

$\mathbf{R}$ F identification (RFID) is a contactless data-capturing technique, which uses RF waves for automatic identification (ID) of objects. The contactless ID system relies on RF waves for data transmission between the data carrying device called the RFID transponder, and the interrogator, which is also known as the RFID reader [1], [2].

Although RFID has found applications in many areas, there is no single RFID system that meets the criteria for every application. The reason for this is that some features are required in some applications, but not others, i.e., some applications require short-range (up to $1.5 \mathrm{~m}$ ) low-cost tags (luggage tagging), while others require long-range (over $20 \mathrm{~m}$ ) and more reliable/robust tags (expensive equipment tagging or vehicle tagging). Hence, the design and choice of RFID systems are application specific.

Significant advance and the lowering of tag costs (below 20 cents) [3] have been achieved within a short period of time due to the adoption of RFID technology by major retail chains like WalMart and the U.S. Department of Defense. Even so, tagging of documents and large volumes of paper/plastic-based

Manuscript received August 20, 2008; revised January 27, 2009. First published April 14, 2009; current version published May 06, 2009. This work was supported in part by the Australian Research Council under Discovery Grant DP0665523: Chipless RFID for Barcode Replacement.

S. Preradovic, I. Balbin, and N. C. Karmakar are with the Department of Electrical and Computer Systems Engineering, Monash University, 3800 Vic., Australia (e-mail: Stevan.Preradovic@ieee.org; Isaac.Balbin@eng.monash.edu.au; Nemai.Karmakar@eng.monash.edu.au).

G. F. Swiegers is with the Security Devices Research Group, Commonwealth Scientific and Industrial Research Organisation (CSIRO) Molecular and Health Technologies, Clayton, Vic. 3169, Australia (e-mail: Gerry.Swiegers@csiro.au).

Color versions of one or more of the figures in this paper are available online at http://ieeexplore.ieee.org.

Digital Object Identifier 10.1109/TMTT.2009.2017323 items such as banknotes, postage stamps, tickets, and envelopes is a problem due to the relatively high price of the tag when compared to the price of the tagged item and the presence of an application-specific integrated circuit (ASIC) chip.

Researchers around the world have been working on developing chipless RFID systems, which seem to be a promising solution for low-cost item tagging [5]. Thus far, the only commercially successful chipless RFID system is developed by RFSAW and it is based on surface acoustic waves (SAWs) [6]. Although, SAW tags are fully functional and could well replace the chipped tags, they do not provide a fully printable solution due to their piezoelectric nature, which could not be applied on banknotes, postage stamps, or other paper/plastic based items.

Fully printable chipless RFID tags have been reported using space-filling curves [7] and capacitively tuned dipoles [8]. The space-filling curves require considerable layout modifications for data encoding, while capacitively tuned dipoles could have undesired parasitic mutual coupling effects and size limitations restricting efficient data encoding.

In this paper, we present a novel fully printable chipless RFID system based on multiresonators and cross-polarized ultra-wideband (UWB) monopole antennas where the tag's unique ID is encoded as a spectral signature. We have reported preliminary results on our system, but only focusing on the proof of concept [13]. The main differences between our system and the ones reported in [7], [8], and [13] are that we encode data in both amplitude and phase, show the possibility of designing a UWB chipless tag, and that the system is not based on radar cross section (RCS) backscattering, but on retransmission of the interrogation signal with the encoded unique spectral ID. The received and transmitted signals are cross-polarized in order to achieve good isolation between the two. Due to these differences, we believe to have achieved less mutual coupling effects, greater number of possible bits, and easier encoding than reported in [7] and [8].

This paper is organized as follows. Section II presents the basic theory of the chipless RFID system. Section III deals with the design of the chipless RFID transponder and the reader antennas. The simulated and measured results of the chipless RFID system are presented in Section IV followed by a conclusion in Section V.

\section{CHIPLESS RFID SySteM THEORY}

As mentioned in Section I, it is imperative to lower the price of the transponder in order to tag low-cost paper/plastic-based items such as banknotes, envelopes, tickets, postage stamps, etc. We present a novel chipless RFID system, which uses 


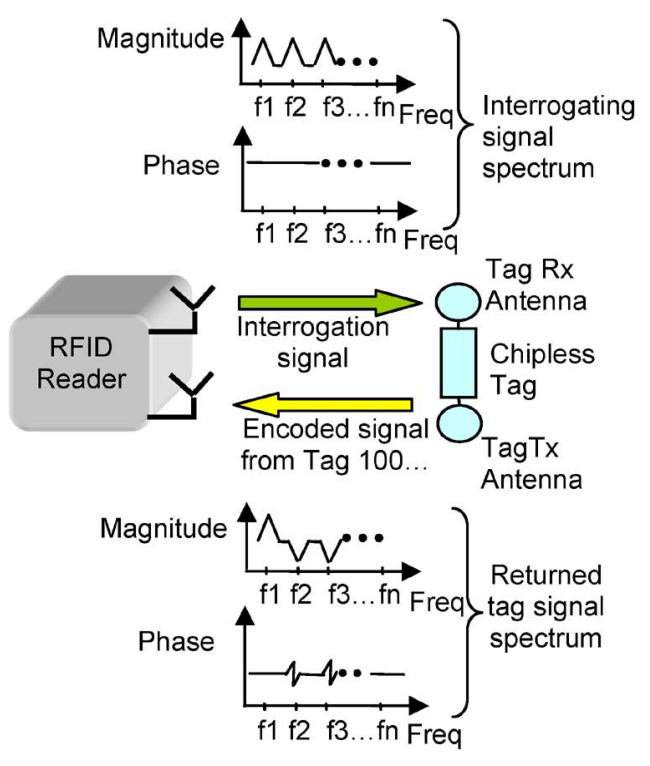

Fig. 1. Physical concept of proposed chipless RFID system.

frequency signatures for data encoding. The proposed chipless RFID system is fully passive and the transponders do not need any power supply in order to operate [9]. The main application for this chipless RFID system is mainly short ranged (up to $40 \mathrm{~cm}$ ) tagging of extremely low-cost items. Hence, power limitation restrictions (transmitted effective isotropic radiated power (EIRP) maximum of $-45-\mathrm{dBm}$ outdoors and $-55-\mathrm{dBm}$ indoors), do not present a major concern for the proposed system. The chipless transponder encodes data in the frequency spectrum; hence, it will have a unique ID or "spectral signature." The spectral signature is obtained by interrogating the transponder by a multifrequency signal. The transponder thus retransmits the received interrogation signal and encodes the data into the frequency spectrum in both magnitude and phase. This allows the reader to use two criteria for data decoding - amplitude and phase. The block diagram of the proposed chipless RFID system is shown in Fig. 1.

The transponder's spectral signature is created with a multiresonating circuit, which is a multistopband filter. The multiresonator is a set of cascaded spiral resonators designed to resonate at particular frequencies and create stopbands. The stopbands introduce attenuation and phase ripple to the transmitted interrogation signal at their resonant frequencies, which can be detected as an amplitude attenuation and phase variation at the reader end. In order to provide isolation between the transmitting and receiving signals, the reader and tag antennas are cross-polarized; hence, becoming practically invisible to each other, but introducing restrictions in transponder's positioning and orientation.

The proposed chipless RFID system is a preliminary design for a short-range conveyor belt system where the tagged items are tracked by moving through the interrogation zone of a fixed reader antenna system, as shown in Fig. 2.

The expected power levels of the received signals from the chipless transponders in an anechoic chamber (loss-less environment) in the antenna far-field region can be calculated using

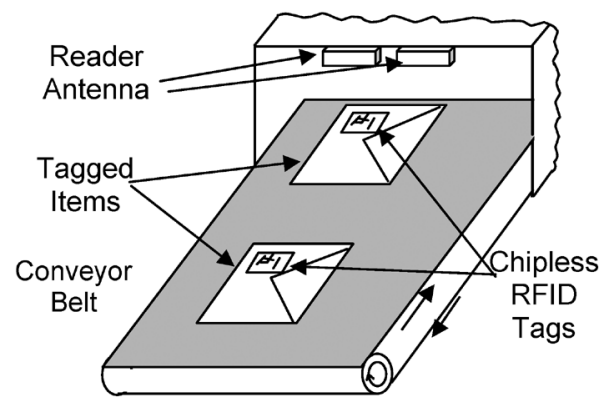

Fig. 2. Potential conveyor belt application for proposed chipless RFID system.

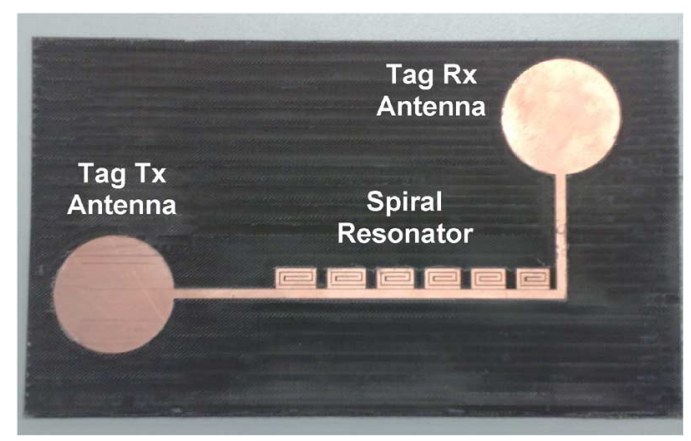

Fig. 3. Photograph of chipless RFID transponder.

the Friis free-space transmission formula [10]. The power density of the signal that reaches the chipless RFID tag in free space is given by

$$
S=\frac{P_{t} G_{r}}{4 \pi r^{2}}
$$

where $P_{t}$ is the transmitted power and $G_{r}$ is the gain of the reader transmitting antenna. The power collected by the transponder's antenna is defined as

$$
P_{a}=S A_{e}=S \frac{\lambda^{2}}{4 \pi} G_{t}
$$

where $A_{e}$ is the effective area of the antenna defined by $G_{t}$, the transponder's antenna gain, and wavelength $\lambda$. Hence, the signal received by the reader after interrogating the transponder is defined as

$$
P_{r x}=\frac{P_{t} G_{t}^{2} G_{r}^{2} \lambda^{4} L(f)}{(4 \pi r)^{4}}
$$

where $L(f)$ is the insertion loss of the transponder's multiresonating circuit as a function of frequency $f$.

Section III presents the design of the chipless RFID transponder, which is comprised of two UWB antennas and a multiresonating circuit and the reader, which is comprised of a broadband log periodic dipole array (LPDA) and transmitting circuit in the form of a vector network analyzer E8361A.

\section{ChiPLess RFID System Design}

\section{A. Chipless RFID Tag Design}

A photograph of the chipless RFID tag manufactured on Taconic TLX-0 $\left(\varepsilon_{r}=2.45, h=0.787 \mathrm{~mm}, \tan \delta=0.0019\right)$ is shown in Fig. 3. It consists of a vertically polarized UWB disc loaded monopole receiving tag antenna, a multiresonating 


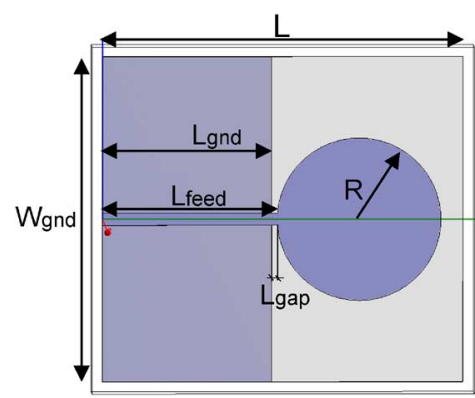

Fig. 4. UWB monopole antenna with defined layout parameters $(L=66 \mathrm{~mm}$, $W_{\text {gnd }}=60 \mathrm{~mm}, L_{\text {gap }}=31 \mathrm{~mm}, L_{\text {feed }}=32 \mathrm{~mm}$, and $R=15 \mathrm{~mm}$, substrate Taconic TLX- $\left.0 \varepsilon_{r}=2.45, h=0.787 \mathrm{~mm}, \tan \delta=0.0019\right)$.

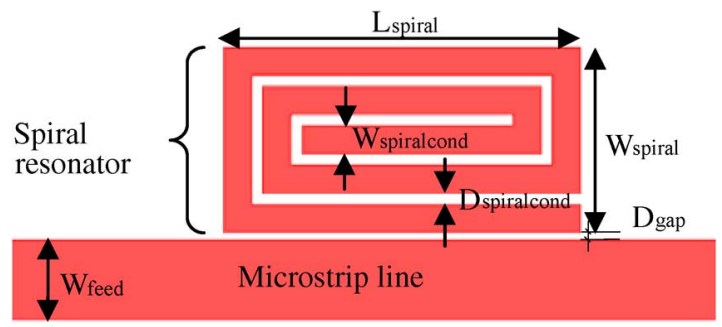

Fig. 5. Layout of a spiral resonator with defined layout parameters.

circuit, and a horizontally polarized UWB transmitting tag antenna. The receiving and transmitting tag antennas are cross-polarized in order to minimize interference between the interrogation signal and the retransmitted encoded signal containing the spectral signature.

The operating frequency bandwidth determines the number of bits that can be used or encoded into a transponder. This is why the chipless transponder requires an antenna with a wide bandwidth and preferably an omnidirectional or wide-beamwidth UWB antenna. Circular UWB monopole antennas have a simple layout and exhibit extremely large bandwidth and a figure-of-eight radiation pattern. The antenna layout parameters are presented in Fig. 4.

The UWB monopole antenna is designed using ADS Momentum on the Taconic TLX-0 substrate. The antenna is optimized to work between $2-2.5 \mathrm{GHz}$.

A multiresonator circuit is used to encode the multifrequency interrogation signal from the reader. The multiresonance was achieved by using cascaded multiple spiral resonators [11] placed next to a microstrip line, as shown in Fig. 5. As can be seen from Fig. 5, $W_{\text {feed }}$ is the width of the microstrip line, $D_{\text {gap }}$ is the separation between the spiral and microstrip line, $W_{\text {spiralcond }}$ is the width of the spiral conductor, $D_{\text {spiralcond }}$ is the separation between the spiral conductors, and $L_{\text {spiral }}$ and $W_{\text {spiral }}$ are the length and width of the spiral resonator. The multiresonator was designed on the Taconic TLX-0 substrate.

Each spiral resonator introduces a different stopband resonance. By varying the dimensions of the spiral resonator, we can vary the resonance. Fig. 6 shows the variation of the spiral resonant frequency with $L_{\text {spiral }}$, as obtained from ADS Momentum simulation.

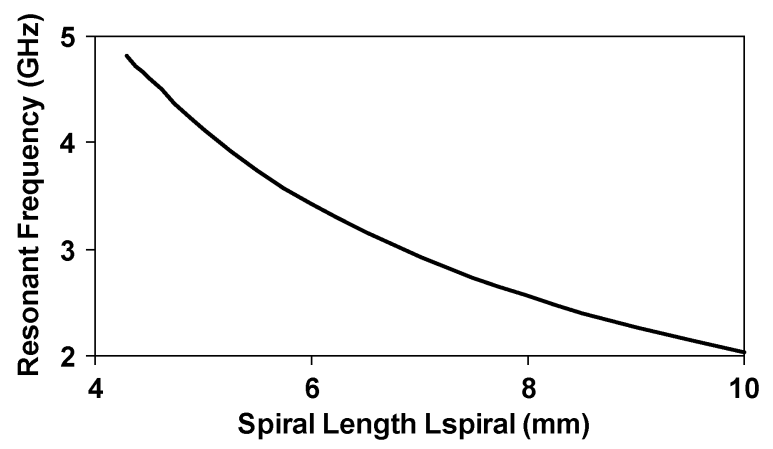

Fig. 6. Variation of the spiral resonant frequency with spiral length $L_{\text {spiral }}$. $W$ feed $=2.26 \mathrm{~mm}, D_{\text {gap }}=0.2 \mathrm{~mm}, D_{\text {spiralcond }}=0.3 \mathrm{~mm}, W_{\text {spiral }}=$ $0.8 \mathrm{~mm}$, substrate Taconic TLX0.

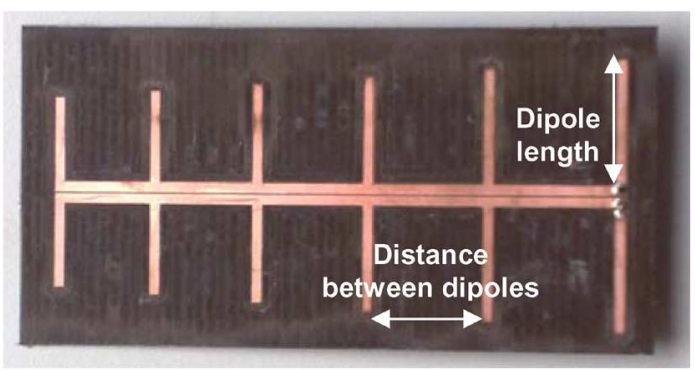

Fig. 7. Photograph of the reader LPDA with defined layout parameters (substrate Taconic TLX-0 $\varepsilon r=2.45, h=0.787 \mathrm{~mm}, \tan \delta=0.0019)$.

TABLE I

LPDA DIMENSIONS

\begin{tabular}{|c|c|}
\hline Dipole length(cm) & Distance between dipoles(cm) \\
\hline 3.22 & 3.22 \\
\hline 2.99 & 2.99 \\
\hline 2.78 & 2.78 \\
\hline 2.58 & 2.58 \\
\hline 2.4 & 2.4 \\
\hline 2.24 & 2.24 \\
\hline
\end{tabular}

\section{B. Chipless RFID Reader Antenna Design}

Directional high gain reader antennas significantly increase the reading range and the number of transponders that are interrogated. LPDA theory and (semiempiric) design equations can be found in [12]. Optimization of the LPDA was done using CST Microwave Studio 2008. Fig. 7 shows a photograph of the designed LPDA on Taconic TLX-0. The layout dimensions of the LPDA are given in Table I.

\section{RESULTS}

A 6-bit chipless RFID tag with six spiral resonators is designed at $2.25 \mathrm{GHz}$. The six bits are represented by six resonant nulls, which are separated approximately by $100 \mathrm{MHz}$ starting from 2 to $2.5 \mathrm{GHz}$. This first design is made to prove the concept of the novel chipless RFID system. In the second design, a UWB 35-bit tag is designed and is presented in Section IV-C. By using the UWB spectrum, we estimate that it is possible to encode over 60 bits into the spectral signature. 


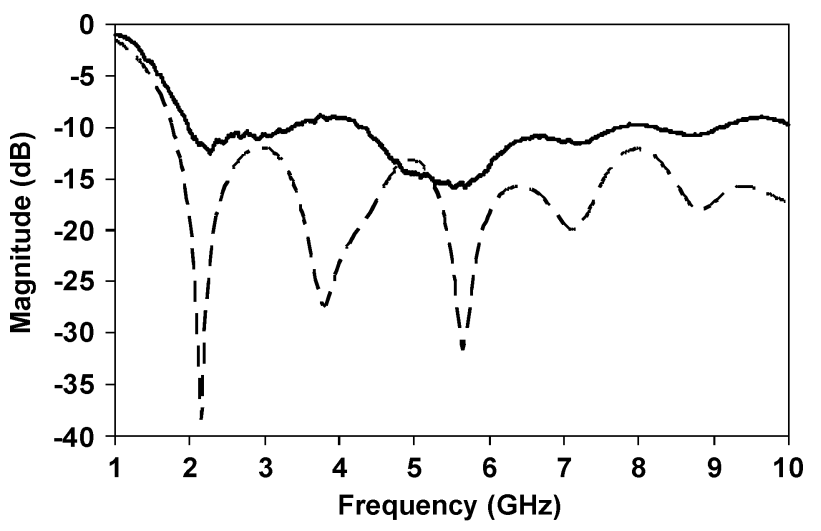

-- Simulated S11 - Measured S11

Fig. 8. Simulated and measured return loss of chipless RFID tag UWB monopole antenna.
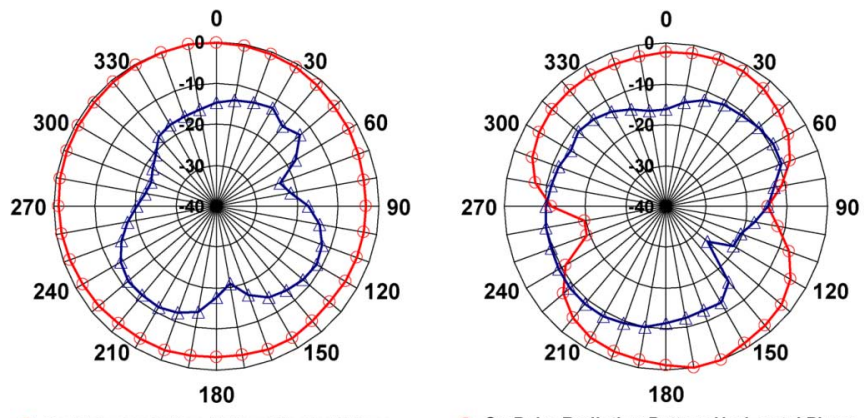

- Co-Polar Radiation Pattern Vertical Plane

- Co-Polar Radiation Pattern Horizontal Plane

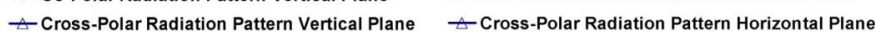

Fig. 9. Measured co-polar and cross-polar radiation patterns of UWB monopole at $2 \mathrm{GHz}$.

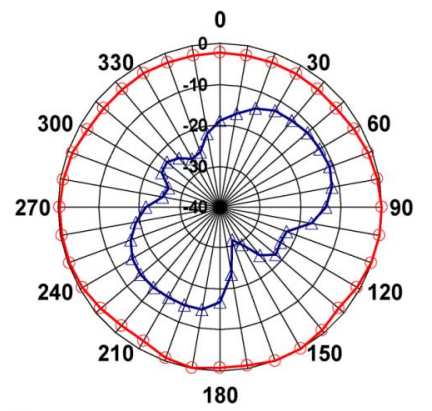

- Co-Polar Radiation Pattern Vertical Plane $\leftarrow$ Cross-Polar Radiation Pattern Vertical Plane

Fig. 10. Measured co-polar and cross-polar radiation patterns of UWB monopole at $2.5 \mathrm{GHz}$.

\section{A. Chipless RFID Tag Antenna}

The simulated and measured return loss versus frequency is shown in Fig. 8. The antenna yields UWB operation with 10-dB return loss from 2 to $10 \mathrm{GHz}$ (up to $10 \mathrm{GHz}$ is measured).

Figs. 9 and 10 show the radiation patterns of the UWB tag antenna at 2 and $2.5 \mathrm{GHz}$ measured inside an anechoic chamber, respectively. As can be seen, the minimum cross-polar level is $-25 \mathrm{~dB}\left(160^{\circ} @ 2-\mathrm{GHz}\right.$ vertical plane $)$ and the maximum is $-7 \mathrm{~dB}\left(90^{\circ} @ 2.5-\mathrm{GHz}\right.$ vertical plane) smaller than the co-polar component. Therefore, the measured radiation patterns ensure polarization purity. The measured antenna gain was $\sim 1 \mathrm{dBi}$

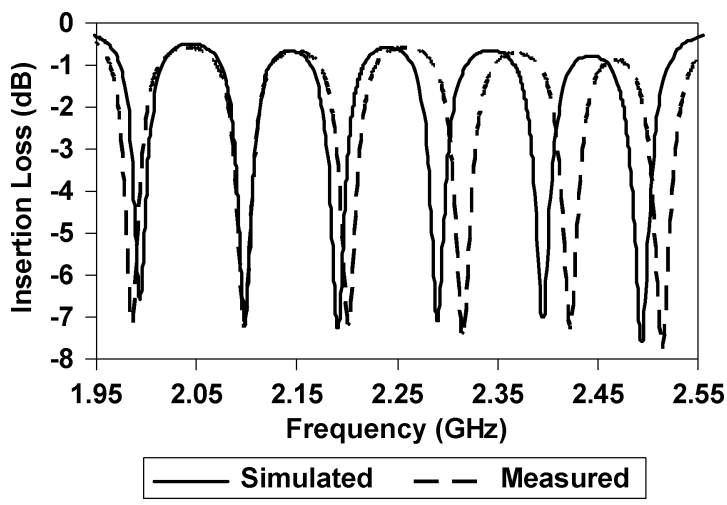

Fig. 11. Simulated and measured results of multiresonator insertion loss.

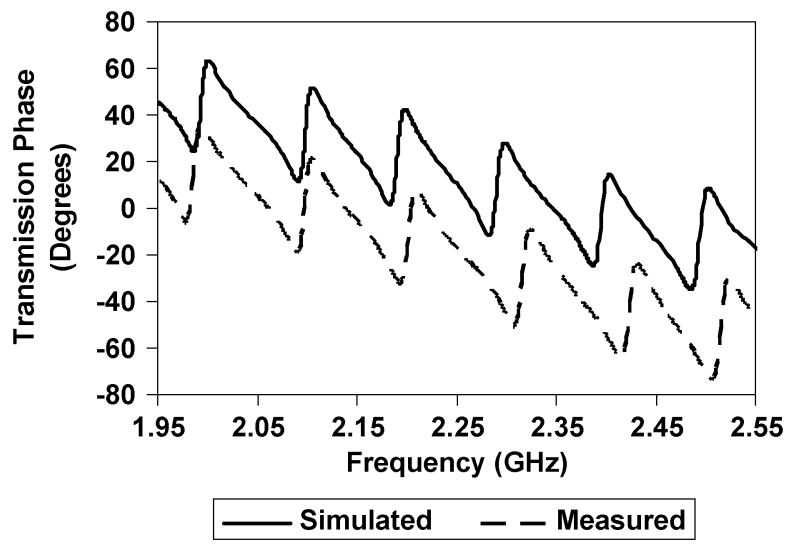

Fig. 12. Simulated and measured results of multiresonator transmission phase.

across the desired frequency band. The suppression of the crosspolar component is essential and can contribute significantly to robust reading results. Hence, we have used reader antennas with better cross-polar suppression than the UWB monopole in order to minimize these unwanted effects.

\section{B. Chipless RFID Tag Multiresonator}

Fig. 11 shows the simulated and measured insertion loss versus frequency, while Fig. 12 shows the simulated and measured transmission phase versus frequency of the multiresonating circuit. The presence of resonant frequency offset in Fig. 11 is due to the printed circuit board (PCB) milling inaccuracies, while the transmission phase offset in Fig. 12 is due to different frequency points created by the simulator when simulating the shorted and unshorted spirals of the multiresonator. The measured two-port $s$-parameter results were obtained using Agilent's PNAE8361A vector network analyzer. From the results shown in Figs. 11 and 12, we can see six distinct resonant nulls in the magnitude and six phase shifts due to the six spirals. Each spiral resonator contributes to a particular resonance, which can be used for data encoding. The phase information can be used as well for data encoding/decoding. The presence of a phase $\left(\sim 40^{\circ}\right)$ ripple at a particular frequency represents logic " 0 ," while the absence of a ripple at a particular frequency represents logic " 1 ."

It is necessary to encode data into the transponder in order for the transponder to have a unique ID. This can be done by 


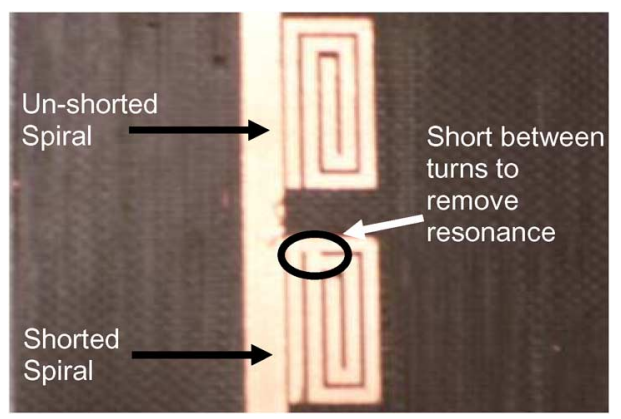

Fig. 13. Photograph of removing spiral resonances via shorting the spiral turn.

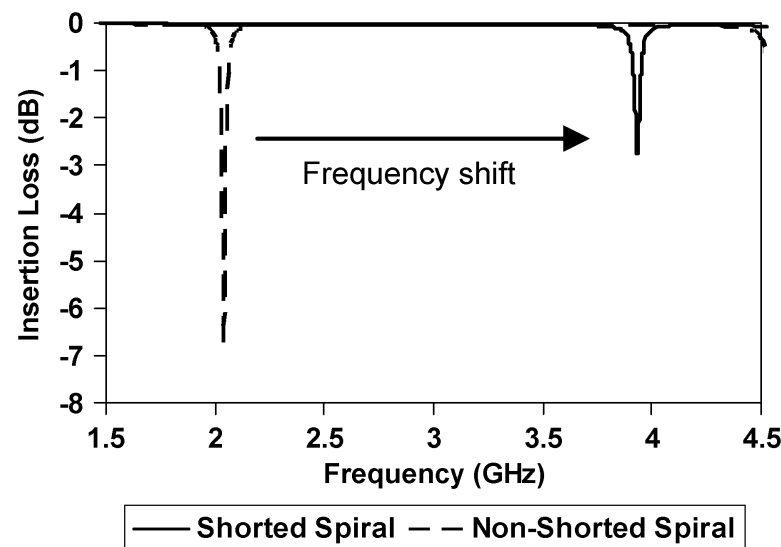

Fig. 14. Frequency shift of resonant frequency with short-circuited spiral.

introducing or removing the resonances of the multiresonator. Fig. 11 shows the $S$-parameter measurements of a multiresonator, which gives a tag ID of 000000. In order to create a different ID, e.g., 101010, the resonances at 2.1, 2.3, and $2.5 \mathrm{GHz}$ need to be removed. By removing the spiral, the resonance is removed as well. The other option is to short the turns of the spiral, as shown in Fig. 13, thus shifting the resonance frequency of the spiral up where it will be of no significance. The shift of the resonant frequency with the shorting of the turns is shown in Fig. 14. The advantage of shorting turns in regard to removing the entire spiral from the layout is the fact that it enables future printing techniques to preserve the layout with all of the spirals shorted, and when encoding data the shorting can be removed via a laser or other etching technique. The frequency signatures of tags with different IDs are shown in Figs. 15 and 16.

Figs. 15 and 16 show the presence of a small frequency shift of the spiral $r$ frequency, which is the result of the change in layout (shorting of the spiral turns) and the error introduced by the manufacturing process done by the PCB milling machine. However, the change in frequency is within an acceptable limit.

\section{UWB 35-bit Chipless RFID Transponder Prototype}

The successful design and results of a 6-bit tag have motivated us to design a 35-bit chipless tag, which may find application for the Australian polymer banknotes and secured documents. We have designed a 35-bit chipless tag operating between 3.1-7 GHz. The photograph of the transponder is shown in Fig. 17.

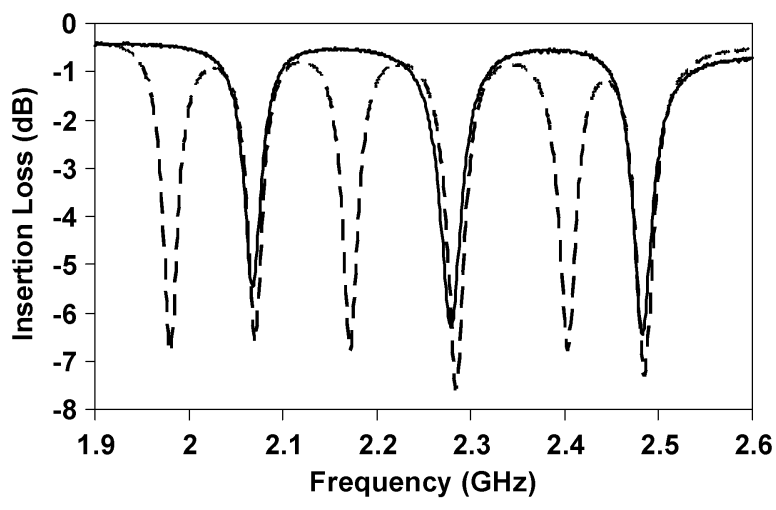

-- Tag 000000ID — Tag101010 ID

Fig. 15. Measured insertion losses of chipless tags with different spectral signatures.

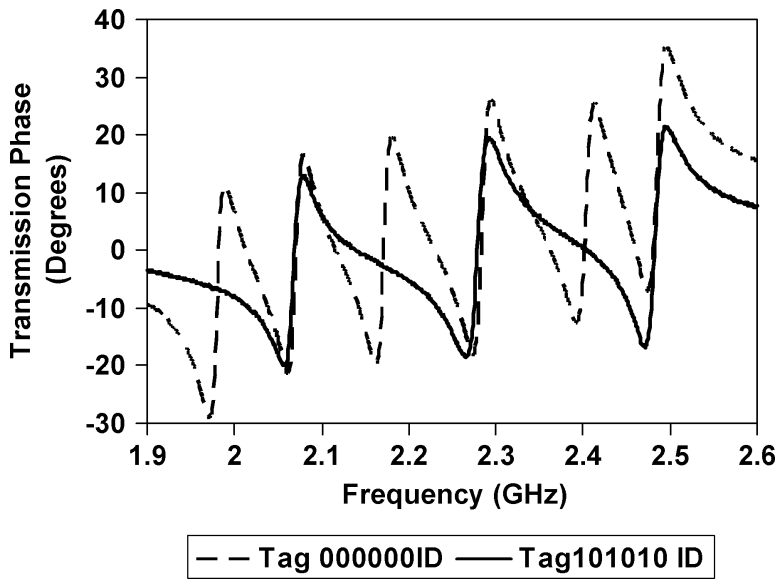

Fig. 16. Measured transmission phase of chipless tags with different spectral signatures.

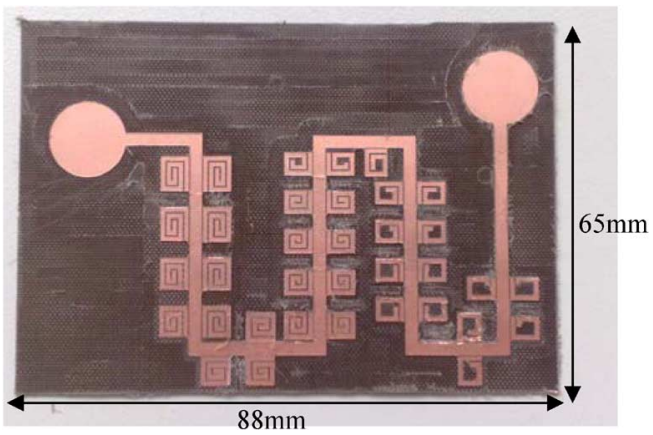

Fig. 17. Photograph of the UWB 35-bit chipless RFID tag with dimensions on Taconic TLX-0 laminate $(\varepsilon r=2.45, h=0.787 \mathrm{~mm}, \tan \delta=0.0019)$.

The chipless transponder section consists of 35 spiral resonators, each having a one-to-one correspondence to a particular bit of data. In order to minimize the size of the transponder, the microstrip line was bent and spiral resonators have been placed on both sides of the microstrip line with 3-mm separation between them. The measured insertion loss of the 35 spiral multiresonator is shown in Fig. 18. The 35 bits of data enable us to have a possible 1.3 billion unique ID combinations. 


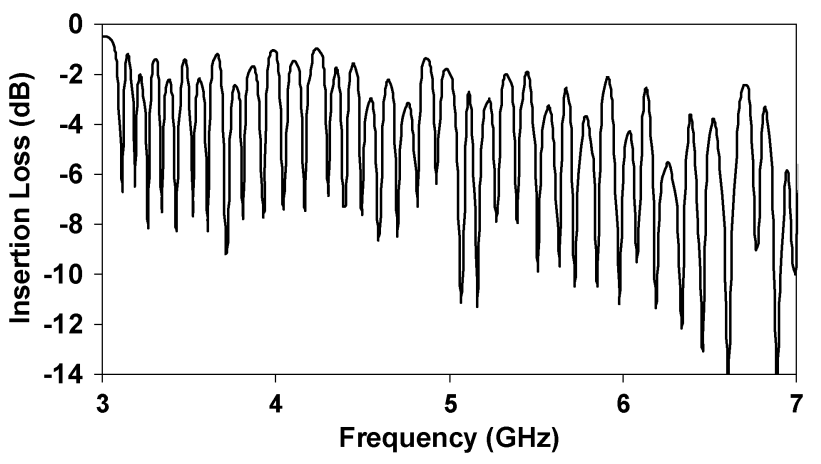

Fig. 18. Measured insertion loss of 35-bit chipless tag multiresonator.

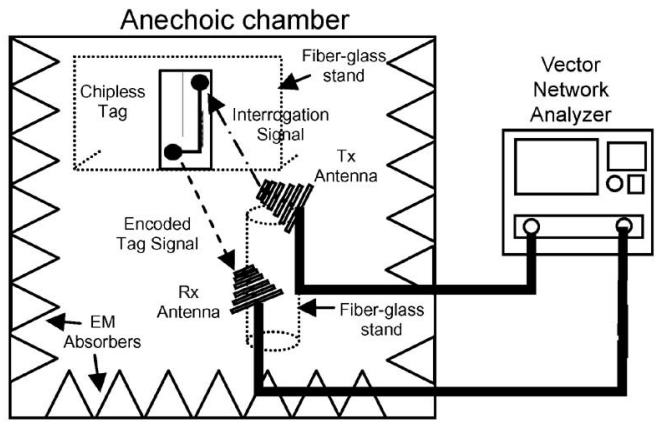

(a)

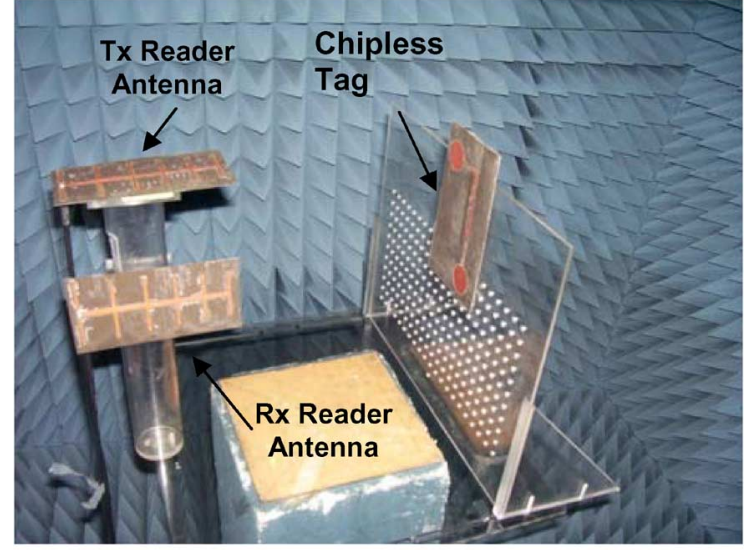

(b)

Fig. 19. Chipless RFID system. (a) Block diagram. (b) Prototype.

\section{Chipless RFID System Field Trials}

The experimental setup is comprised of the LPDA built on Taconic TLX-0 and the vector network analyzer E8361A as the reader electronics and chipless transponder. The experiment was done in an anechoic chamber in order to validate the successful encoding of the transponder and its detection at the reader end using the network analyzer. The chipless transponder along with the reader antennas have been mounted on Fiberglass stands and placed into the anechoic chamber. The block diagram and photograph of the experimental setup are shown in Fig. 19.

Fig. 19 shows that the reader antennas are cross-polarized in order to minimize crosstalk between the two antennas, thus improving isolation of the tag signal. Fig. 20 shows the mea-

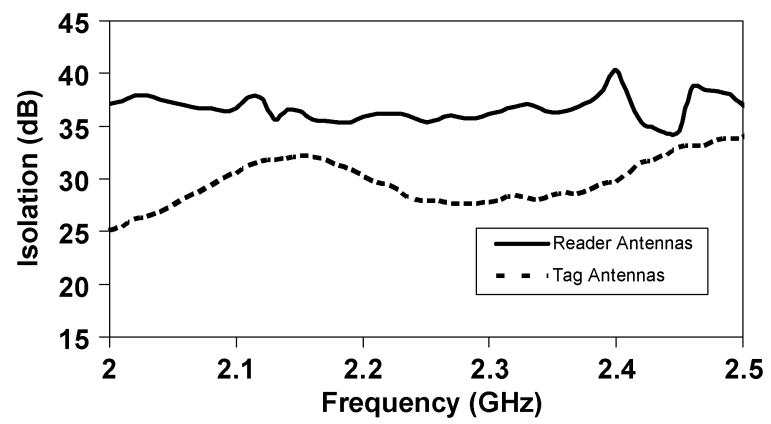

Fig. 20. Measured isolation between cross-polarized reader antennas.

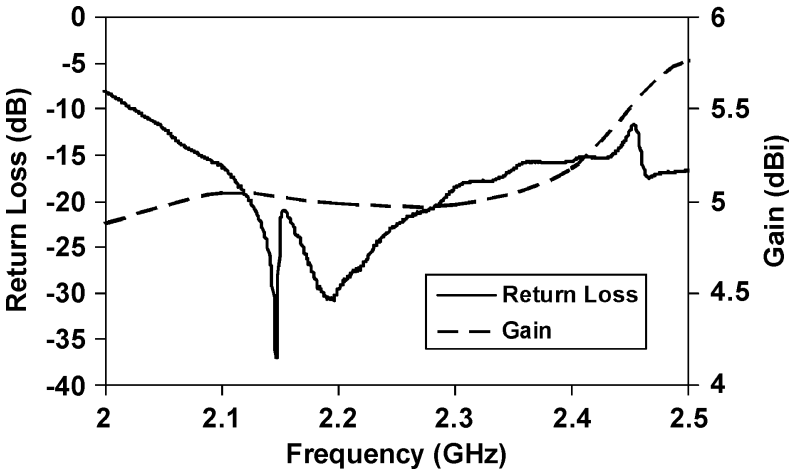

Fig. 21. Measured return loss and gain of LPDA reader antenna.

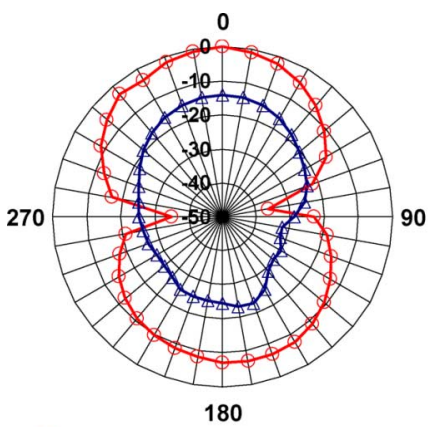

$\smile$ Co-Polar Radiation Pattern at $2 \mathrm{GHz}$ $\triangle$ Cross-Polar Radiation Pattern at $2 \mathrm{GHz}$

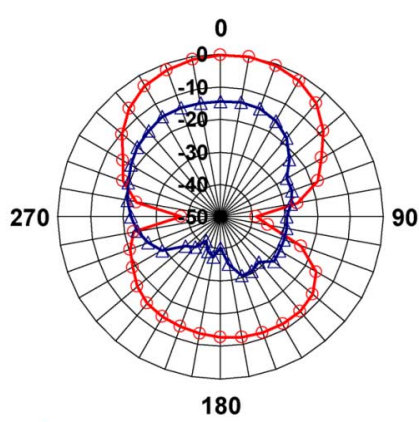

- Co-Polar Radiation Pattern at 2.5GHz $\triangle$ Cross-Polar Radiation Pattern at $2.5 \mathrm{GHz}$
Fig. 22. Measured near-field co-polar and cross-polar radiation patterns of LPDA reader antenna in horizontal plane (18-cm distance).

sured isolation of the omnidirectional UWB monopole tag antennas and directive reader LPDA. From Fig. 20, it is clear that the LPDAs provide greater isolation than the omnidirectional monopoles between $2-2.5 \mathrm{GHz}$.

The measured return loss and gain versus frequency and co-polar and cross-polar radiation patterns in both vertical and horizontal planes in near- and far-field conditions are shown in Figs. 21-25, respectively. The antenna measurements were performed in an anechoic chamber. The near-field radiation patterns are of particular interest due to the fact that the proposed tag is read mostly in the near field of the LPDA reader antenna. As can be seen from Figs. 22 and 23, the polarization purity in the near-field region is still intact with more than 10-dB cross-polar levels.

The LPDA is a preferred candidate for the reader antenna to the UWB monopoles due to better isolation between the two 


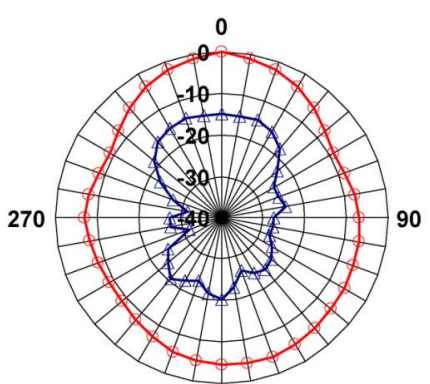

180

- Co-Polar Radiation Pattern at $2 \mathrm{GHz}$

$\triangle$ Cross-Polar Radiation Pattern at $2 \mathrm{GHz}$

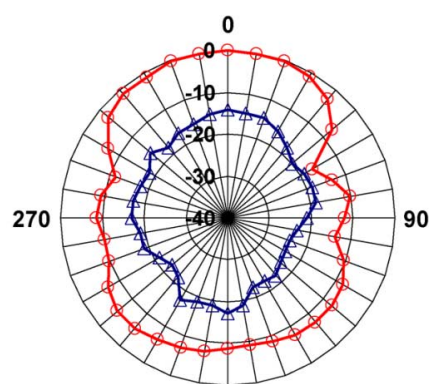

180

-Co-Polar Radiation Pattern at 2.5 $\mathrm{GHz}$ $\triangle$ Cross-Polar Radiation Pattern at $2.5 \mathrm{GHz}$

Fig. 23. Measured near-field co-polar and cross-polar radiation patterns of LPDA reader antenna in vertical plane (18-cm distance).

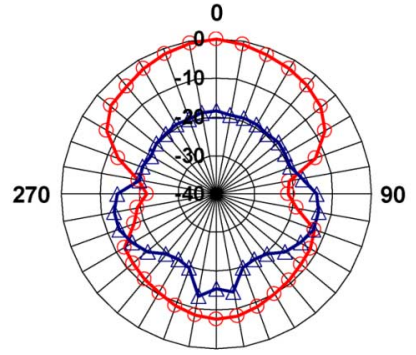

180

- Co-Polar Radiation Pattern @ 2GHz

$\triangle$ Cross-Polar Radiation Pattern @ 2GHz

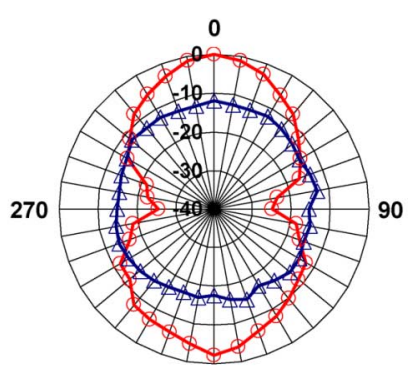

180

- Co-Polar Radiation Pattern @ 2.5 GHz

$\triangle$ Cross-Polar Radiation Pattern @ 2.5 GHz

Fig. 24. Measured far-field co-polar and cross-polar radiation patterns of LPDA reader antenna in horizontal plane (86- $\mathrm{cm}$ distance).

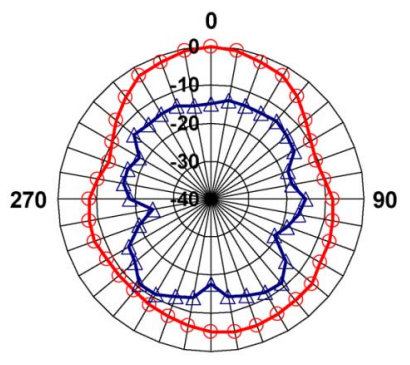

180

- Co-Polar Radiation Pattern @ 2GHz $\_$Cross-Polar Radiation Pattern @ 2GHz

Fig. 25. Measured far-field co-polar and cross-polar radiation patterns of LPDA reader antenna in vertical plane (86-cm distance).

cross-polarized antennas (Fig. 20), more directive radiation pattern, which creates a stronger line-of-sight (LOS) component, higher gain, and its low cross-polar components.

We encoded the transponder with ID 000000 and placed it from 5 to $40 \mathrm{~cm}$ (in steps of $5 \mathrm{~cm}$ ) away from the LPDA reader antennas, as shown in Fig. 19. The same experiment was conducted using the UWB monopoles as reader antennas, but a reading range of only a couple of centimeters was achieved. The measured results of the received data are presented in both magnitude and phase with the variation of the tag to reader distance. The Agilent E8361A network analyzer was used in the measurement. The analyzer was calibrated with the output power of the ports being $-28 \mathrm{dBm}$.

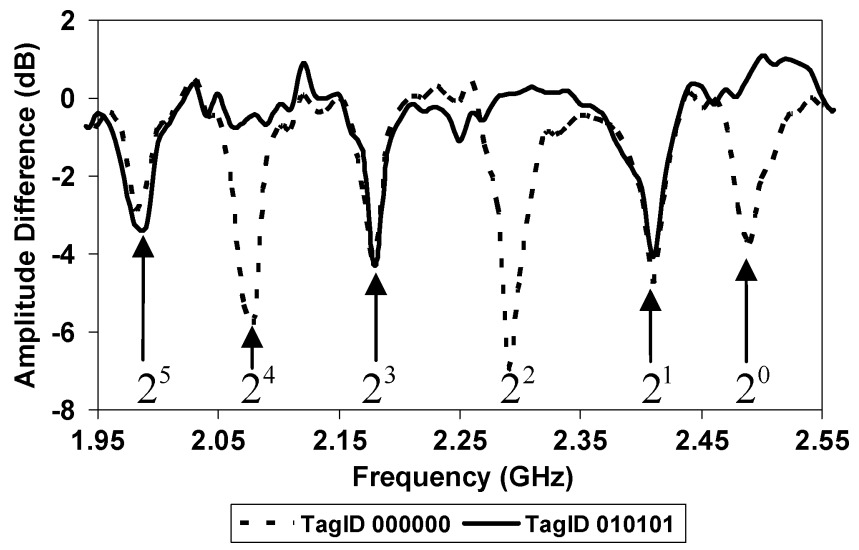

Fig. 26. Amplitude variations of the received tag signal at the reader end for chipless transponders at $5 \mathrm{~cm}$.

The amplitude information in the spectral signature was measured and assessed in such a way that the tag with the 111111 ID is used as a reference. The amplitude difference of the received power due to the transponder frequency signatures is presented in Fig. 26 and Table II. Fig. 27 shows the amplitude difference in respect to the 111111 ID of the received signal after interrogating the same transponder rotated by $180^{\circ}$, hence creating a polarization mismatch. The amplitude information is considered completely unreadable and, therefore, useless.

Table II shows that the transponder readability drops with the increase of the transponder's distance. The transponder was successfully read with LPDA at distances up to $10 \mathrm{~cm}$, but reading ranges over $10 \mathrm{~cm}$ created errors mostly for $2^{5}$ most significant bit, which has the resonant null at $\sim 2 \mathrm{GHz}$. These errors can be attributed to the high cross-polar level of the tag's monopole and impedance mismatch of both tag and reader antennas at that frequency. An optimization of the design should rectify this problem.

The experiment was performed within the near- and near farfield of the reader antennas (far-field is $38.4 \mathrm{~cm}$ at $2.25 \mathrm{GHz}$ using $2 d^{2} / \lambda$, where $d$ is the antenna largest dimension, in this case, $16 \mathrm{~cm}$, and $\lambda$ is the wavelength). Hence, the system is designed to operate in both near- and near far-field conditions.

The phase information in the spectral signature was measured and assessed in such a way that the tag with the 111111 ID is used as a reference. The variation of the received power phase due to the transponder frequency signatures is presented in Fig. 28 and Table II. Fig. 29 shows the phase information when the transponder is rotated by $180^{\circ}$ creating complete polarization mismatch with the reader antennas. The distinct phase difference between the two states is completely unusable.

From Table II, we can see that the transponder is accurately read by the reader (even at $40 \mathrm{~cm}$, which is the far-field), thus we can conclude that the phase information is more resilient to noise and can be read from a greater distance when compared to the amplitude information of the frequency signature. This represents a novelty in data encoding and data extraction when it comes to chipless frequency-signature-based RFID systems. Other frequency-signature-based chipless RFID systems are based on examining and encoding the magnitude of the signal [7], [8], while this system encodes data into both phase and 
TABLE II

Chipless Tag Reading Results Between Tag 000000 and Tag $111111 \Delta$ A: AMPLITUdE DifFERENCE IN DECIBElS, $\Delta \Theta$ : PHASE DiFFERENCE IN DEGREES

\begin{tabular}{|c|c|c|c|c|c|c|c|c|c|c|c|c|}
\hline \multirow[t]{2}{*}{ Distance $(\mathrm{cm})$} & \multicolumn{2}{|c|}{$2^{5}$} & \multicolumn{2}{|c|}{$2^{4}$} & \multicolumn{2}{|c|}{$2^{3}$} & \multicolumn{2}{|c|}{$2^{2}$} & \multicolumn{2}{|c|}{$2^{1}$} & \multicolumn{2}{|c|}{$2^{0}$} \\
\hline & $\Delta \mathrm{A}$ & $\Delta \theta$ & $\Delta \mathrm{A}$ & $\Delta \theta$ & $\Delta \mathrm{A}$ & $\Delta \theta$ & $\Delta \mathrm{A}$ & $\Delta \theta$ & $\Delta \mathrm{A}$ & $\Delta \theta$ & $\Delta \mathrm{A}$ & $\Delta \theta$ \\
\hline 5 & -3 & 14 & -6 & 16 & -4 & 17 & -7 & 17 & -4 & 17 & -4 & 18 \\
\hline 10 & -1 & 15 & -2 & 40 & -3 & 30 & -2 & 25 & -1 & 15 & -2 & 42 \\
\hline 15 & 0 & 14 & -1 & 18 & -1 & 28 & -1 & 16 & -1 & 25 & -1 & 15 \\
\hline 20 & 0 & 12 & -1 & 14 & -2 & 17 & -3 & 16 & -1 & 26 & -1 & 26 \\
\hline 25 & 0 & 12 & -4 & 16 & -4 & 24 & -3 & 22 & -1 & 14 & -3 & 23 \\
\hline 30 & 0 & 10 & 0 & 18 & -1 & 15 & 0 & 15 & -1 & 21 & -2 & 30 \\
\hline 40 & 0 & 7 & 0 & 9 & 0 & 6 & -1 & 12 & 0 & 12 & -1 & 10 \\
\hline
\end{tabular}

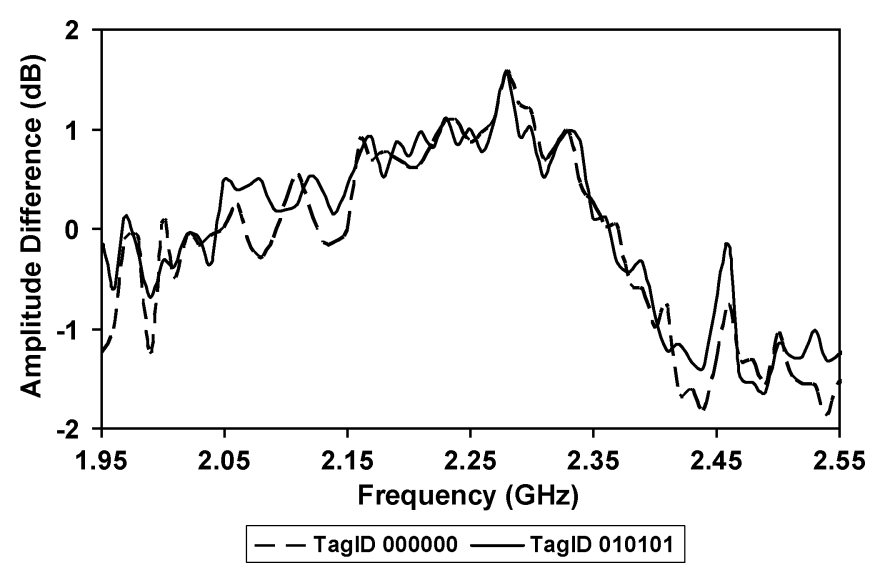

Fig. 27. Received signals by reader for chipless transponders at $5 \mathrm{~cm}$ rotated by $180^{\circ}$ (polarization mismatch between tag and reader antennas).

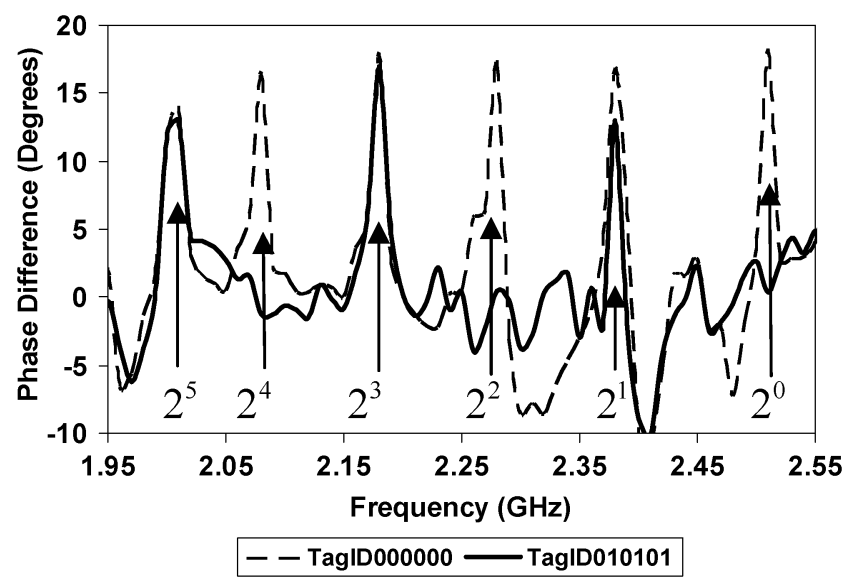

Fig. 28. Phase variations of the received tag signal at the reader end for chipless transponders at $5 \mathrm{~cm}$.

magnitude, thus enabling complete information for decoding the frequency signature (magnitude and phase) where phase is preferred for greater reading ranges and accuracy.

\section{CONCLUSION}

In this paper, we have presented a novel fully printable chipless RFID system, which can be used for tracking low-cost items such as note bills, envelopes, and other paper/plastic products, items, and documents. The operating system uses multiresonators to encode data into the spectral signature. By interrogating the transponder by a multifrequency signal, it is

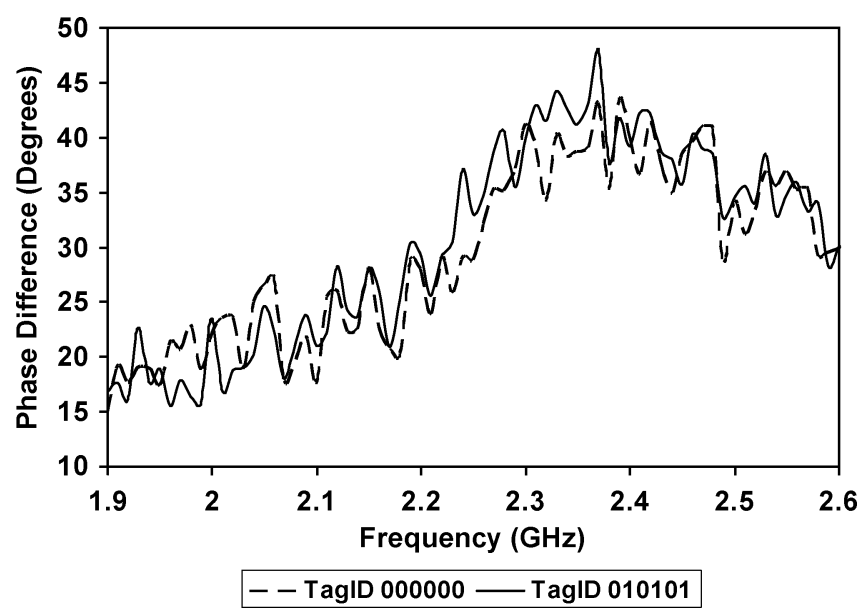

Fig. 29. Phase variations of the received tag signal at the reader end for chipless transponders at $5 \mathrm{~cm}$ rotated by $180^{\circ}$ (wrong alignment).

possible to detect the variations in the magnitude and phase of the received tag signal and decode the transponders ID. The simulation and experimental results validate the successful design of the chipless RFID concept and prototype tag. The simple shorting of spiral turns shifts the resonant frequency of the spiral to an insignificant frequency, which is not used in the system. The frequency-signature-based system is the first of its kind to use phase to encode data, which increases the reading range and accuracy and provides the reader with the ability to interrogate magnitude and phase and compare them to confirm the ID of the interrogated tag. It is necessary to use a reference signature ID of 111111 when performing amplitude and phase data decoding. The system has a great potential if printed on a plastic substrate using transparent conductive (silver) ink for low-cost item tracking. It would represent a cheap and economical way of potentially replacing the barcode due to the fact that the chipless transponders are not comprised of any silicon-based circuits or semiconductor devices. The UWB frequency band would allow the transponder to encode over 60 bits. Future research will be concentrated on better accuracy of reading, developing the chipless system on plastic substrates, and RFID reader development with anticollision protocols.

\section{REFERENCES}

[1] K. Finkenzeller, RFID Handbook, 2nd ed. Munich, Germany: Wiley, 2003

[2] U. Kraiser and W. Steinhagen, "A low-power transponder IC for highperformance identification systems," IEEE J. Solid-State Circuits, vol. 30, no. 3, pp. 306-310, Mar. 1995. 
[3] P. Harrop, "The price-sensitivity curve for RFID," IDTechEx, Cambridge, U.K., Internet article, Aug. 2006. [Online]. Available: http:// www.idtechex.com/products/en/articles/00000488.asp, (accessed May 2008).

[4] J. Collins, "Alien cuts tag price," RFID J. April 2004. [Online]. Available: http://www.rfidjournal.com/article/articleview/857/1/1/, (accessed Apr. 2008).

[5] R. Das, "Chipless RFID-The End Game," IDTechEx, Cambridge, MA, Internet article, Feb. 2006. [Online]. Available: http://www. idtechex.com/products/en/articles/00000435.asp, (accessed Apr. 2008).

[6] C. S. Hartmann, "A global SAW ID tag with large data capacity," in Proc. IEEE Ultrason. Symp., Munich, Germany, October 2002, vol. 1, pp. 65-69. [Online]. Available: http://www.rfsaw.com/pdfs/ Global_SAW_ID_Tag_lg.pdf, reprint form.

[7] J. McVay, A. Hoorfar, and N. Engheta, "Space-filling curve RFID tags," in IEEE Radio Wireless Symp., San Diego, CA, Jan. 17-19, 2006, pp. 199-202.

[8] I. Jalaly and D. Robertson, "Capacitively-tuned split microstrip resonators for RFID barcodes," in Eur. Microw. Conf., Paris, France, Oct. 4-6, 2005, vol. 2, pp. 4-7.

[9] U. Karthaus and M. Fischer, "Fully integrated passive UHF RFID transponder IC with 16.7- $\mu \mathrm{W}$ minimum RF input power," IEEE $J$. Solid-State Circuits, vol. 38, no. 10, pp. 1602-1608, Oct. 2003.

[10] K. V. S. Rao, P. V. Nikitin, and S. M. Lam, "Antenna design for UHF RFID tags: A review and a practical application," IEEE Trans. Antennas Propag., vol. 53, no. 12, pp. 3870-3876, Dec. 2005.

[11] J. Joubert, "Spiral microstrip resonators for narrow-Stopband filters," Proc. Inst. Elect. Eng.-Microw., Antennas, Propag., vol. 150, no. 6, pp. 493-496, Dec. 2003.

[12] C. A. Balanis, Antenna Theory: Analysis and Design, 2nd ed. New York: Wiley, 1982.

[13] S. Preradovic, I. Balbin, N. C. Karmakar, and G. Swiegers, "A novel chipless RFID system based on planar multiresonators for barcode replacement," in IEEE Int. RFID Conf., Las Vegas, NV, Apr. 16-17, 2008, pp. 289-296.

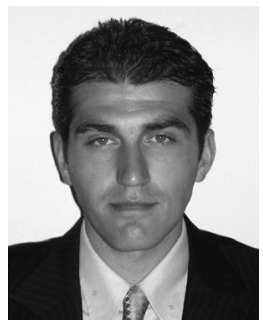

Stevan Preradovic (S'08) was born in Sombor, Serbia. He received the Bachelor of Engineering degree in microcomputer electronics from the University of Novi Sad, Novi Sad, Serbia, in 2005, and is currently working toward the Ph.D. degree in electrical and computer systems engineering at Monash University, Melbourne, Vic., Australia. He has authored several journal publications and a number of conference papers. His research interests include design of chipless RFID systems and readers, $\mathrm{RF} /$ microwave filters, and embedded systems.

Mr. Preradovic was the recipient of First Prize at the student challenge competition of European Microwave Week (EUMW) 2008.

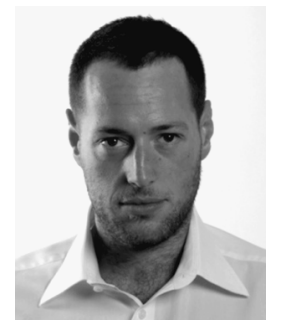

Isaac Balbin ( $\left.\mathrm{S}^{\prime} 07\right)$ received the Bachelor of Engineering (B.E.) and Bachelor of Science (B.Sc.) degrees from Monash University, Melbourne, Vic., Australia in 2005, and is currently working toward the Ph.D. degree in engineering at Monash University. His thesis was based on negative refractive index metamaterials.

His research interests include passive microstrip technology, chipless RFID systems, short-range radar applications, innovative antenna design based on metamaterials, and UWB circuits and systems.

Mr. Balbin was given the top mark for the year for his thesis, as well as the Commonwealth Scientific and Industrial Research Organisation (CSIRO) Prize for best thesis in telecommunications and the Monash University Siemens nomination for innovation.

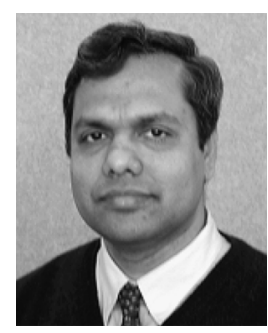

Nemai Chandra Karmakar (S'91-M'91-SM'99) received the M.Sc. degree in electrical engineering from the University of Saskatchewan, Saskatoon, SK, Canada, in 1991, and the Ph.D. degree from the University of Queensland, Brisbane, Qld., Australia in 1999.

$\mathrm{He}$ is a Senior Lecturer with the Department of Electrical and Computer Systems Engineering, Monash University, Clayton, Vic., Australia. He possesses approximately 18 years of teaching, design, and development experience in antennas, microwave active and passive circuits, and RFIDs in Canada, Australia, and Singapore. He has authored or coauthored over 180 referred journal and conference papers, nine book chapters, and is currently preparing one book. He holds two patents.

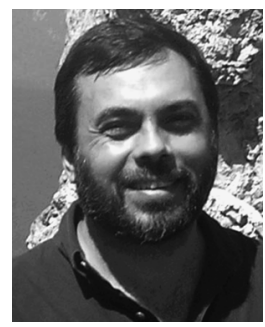

Gerhard (Gerry) F. Swiegers is a Leader of the Security Devices Research Group, Commonwealth Scientific and Industrial Research Organisation (CSIRO), Molecular and Health Technology, the division of CSIRO that developed the all-polymer banknote in collaboration with the Reserve Bank of Australia. Polymer banknotes of this type are now used in over 22 countries worldwide, with more than four billion notes produced to date. He has authored or coauthored over 50 peer-reviewed publications including five book chapters. He holds 11 patents.

His research interests and activities involve a diverse and eclectic range of topics in chemistry and physics. 